\title{
Structure of states which satisfy strong subadditivity of quantum entropy with equality
}

\author{
Patrick Hayder $*$ \\ Institute for Quantum Information, Caltech 107-81, Pasadena, CA 91125, USA \\ Richard Jozsd \\ Department of Computer Science, University of Bristol, \\ Merchant Venturers Building, Woodland Road, Bristol BS8 1UB, United Kingdom \\ Dénes Pett \\ Department for Mathematical Analysis, Mathematical Institute, \\ Budapest University of Technology and Economics, Egry József utca 2, H-1111 Budapest, Hungary \\ Andreas Winter $\$$ \\ Department of Computer Science, University of Bristol, \\ Merchant Venturers Building, Woodland Rd, Bristol BS8 1UB, United Kingdom
}

(Dated: 22nd August 2003)

\begin{abstract}
We give an explicit characterisation of the quantum states which saturate the strong subadditivity inequality for the von Neumann entropy. By combining a result of Petz characterising the equality case for the monotonicity of relative entropy with a recent theorem by Koashi and Imoto, we show that such states will have the form of a so-called short quantum Markov chain, which in turn implies that two of the systems are independent conditioned on the third, in a physically meaningful sense. This characterisation simultaneously generalises known necessary and sufficient entropic conditions for quantum error correction as well as the conditions for the achievability of the Holevo bound on accessible information.
\end{abstract}

PACS numbers: 03.65.Ta, 03.67.Hk

Keywords: Entropy, strong subadditivity, invariant algebras, quantum Markov state

\section{INTRODUCTION}

The von Neumann entropy [13]

$$
S(\rho)=-\operatorname{Tr} \rho \log \rho,
$$

of a density operator $\rho$ on a finite dimensional Hilbert space $\mathcal{H}$ shares many properties with its classical counterpart, the Shannon entropy

$$
H(P)=-\sum_{x \in \mathcal{X}} P(x) \log P(x)
$$

of a probability distribution $P$ on a discrete set $\mathcal{X}$. (All logarithms in this work are understood to be to base 2 . Also, we will use the terms "state" and "density operator" interchangeably.) For example, both are nonnegative, and equal to 0 if and only if the state (distribution) is an extreme point in the set of all states (distributions), i.e. if $\rho$ is pure ( $P$ is a point mass). Both are concave and, moreover, both are subadditive: for a state $\rho_{A B}$ on a composite system $\mathcal{H}_{A} \otimes \mathcal{H}_{B}$ with reduced states

$$
\rho_{A}=\operatorname{Tr}_{B}\left(\rho_{A B}\right), \quad \rho_{B}=\operatorname{Tr}_{A}\left(\rho_{A B}\right),
$$

\footnotetext{
*Electronic address: patrick@cs.caltech.edu

$\dagger$ Electronic address: richard@cs.bris.ac.uk

‡Electronic address: petz@math.bme.hu

$\S$ Electronic address: winter@cs.bris.ac.uk
}

it holds that

$$
S\left(\rho_{A B}\right) \leq S\left(\rho_{A}\right)+S\left(\rho_{B}\right) .
$$

A directly analogous inequality holds for a distribution over a product set and its marginals. (Many more properties of $S$ are collected in the review by Wehrl 26] and in the monograph [14].)

We shall view von Neumann entropy as a generalisation of Shannon entropy [19] in the following precise way: if the set $\mathcal{X}$ labels an orthonormal basis $\{|x\rangle: x \in \mathcal{X}\}$ of $\mathcal{H}_{X}$ we can construct the state

$$
\rho_{P}=\sum_{x} P(x)|x\rangle\langle x|
$$

corresponding to the distribution $P$. This clearly defines an affine linear map from distributions into states. It is then straightforward to check that

$$
S\left(\rho_{P}\right)=H(P),
$$

so all properties of von Neumann entropy of a single system also hold for Shannon entropy of a single distribution.

Similarly, for a distribution $P$ on a cartesian product $\mathcal{X} \times \mathcal{Y}$, we use the tensor product basis

$$
\{|x y\rangle=|x\rangle \otimes|y\rangle: x \in \mathcal{X}, y \in \mathcal{Y}\}
$$


to define the state $\rho_{P}$ on $\mathcal{H}_{X} \otimes \mathcal{H}_{Y}$. Again, it is straightforward to check that reduced states correspond to taking marginals:

$$
\operatorname{Tr}_{Y}\left(\rho_{P}\right)=\rho_{P \mid \mathcal{X}}, \quad \operatorname{Tr}_{X}\left(\rho_{P}\right)=\rho_{P \mid \mathcal{Y}} .
$$

Hence all entropy relations for bipartite states also hold for bipartite distributions.

In [9] Lieb and Ruskai proved the remarkable relation

$$
S\left(\rho_{A B}\right)+S\left(\rho_{B C}\right) \geq S\left(\rho_{A B C}\right)+S\left(\rho_{B}\right),
$$

with a tripartite state $\rho_{A B C}$ on the system $\mathcal{H}_{A} \otimes \mathcal{H}_{B} \otimes \mathcal{H}_{C}$. It clearly generalises the previous subadditivity relation, which is recovered for a trivial system $B: \mathcal{H}_{B}=\mathbb{C}$. In fact, this inequality plays a crucial role in nearly every nontrivial insight in quantum information theory, from the famous Holevo bound [5] and the properties of the coherent information [3, 18] to the recently proved additivity of capacity for entanglement-breaking channels [20].

The present investigation aims to resolve the problem of characterising the states which satisfy this relation with equality: the main result is theorem [6] Roughly speaking, the strong subadditivity inequality expresses the fact that discarding a subsystem of a quantum system is a dissipative operation, in the sense that it can only destroy correlations with the rest of the world. Our work, therefore, can be interpreted as providing a detailed description of the conditions under which the act of discarding a quantum system can be locally reversed on a particular input. We restrict ourselves to finite dimensional systems in this paper. The question of whether a similar result holds in infinite dimension is left open.

The rest of the paper is organised as follows. In section [I] we will review the case of probability distributions: there the solution to our problem is easy to obtain, and in fact well-known. This will provide the intuitive basis for understanding our main result. After that, in section III we review quantum relative entropy and the relation of its monotonicity property to the strong subadditivity inequality. Section IV presents a condition given by Petz for equality in the monotonicity of relative entropy, while section $\nabla$ presents and proves our main result, a structure theorem for states which satisfy strong subadditivity with equality. An essential step is the application of a recent result of Koashi and Imoto 7] for which we give a short but non-constructive algebraic proof in the appendix. In section VI we show how the entropic conditions for quantum error correction as well as the conditions for saturation in the Holevo bound follow as easy corollaries from our structure theorem.

The question of characterising the equality case of strong subadditivity as well as of the monotonicity of relative entropy was considered in earlier work by Petz 15], where it was related to the existence of quantum operations with certain properties. Ruskai [17] has given a characterisation in terms of an operator equality, which can be used to show that the states described in our main theorem [6] are indeed equality cases (as she has informed us, this was pointed out to her by M. A. Nielsen after [17] appeared). Neither of these results is as explicit as one could wish for, however, because while both give algebraic criteria which one can check on any given state, they do not yield a simple description of all the states that satisfy equality. This simple description is exactly what our theorem [6] provides.

\section{THE CLASSICAL CASE}

Let us first look at the classical case of probability distributions and their Shannon entropies. The exposition is most conveniently phrased in terms of random variables denoted $A, B, C$, taking values in $\mathcal{A}, \mathcal{B}, \mathcal{C}$, respectively, with a joint distribution

$$
P_{A B C}(a, b, c)=\operatorname{Pr}\{A=a, B=b, C=c\} .
$$

The distribution of $A$ is the marginal $P_{A}=\left.P_{A B C}\right|_{\mathcal{A}}$ of the joint distribution to $\mathcal{A}$ and similarly for the other variables.

Shannon [19] defined the mutual information

$$
I(A: B)=H(A)+H(B)-H(A B),
$$

with $H(A)=H\left(P_{A}\right)$ and so on. It is not hard to show that $I(A: B) \geq 0$ with equality if and only if $A$ and $B$ are independent.

Conditional mutual information is defined as

$$
I(A: C \mid B)=\sum_{b \in \mathcal{B}} P_{B}(b) I(A: C \mid B=b),
$$

where $I(A: C \mid B=b)$ is the mutual information between the variables $A$ and $C$ conditional on the event " $B=b$ ", i.e. $I\left(\left.A\right|_{B=b}:\left.C\right|_{B=b}\right)$, with

$$
\begin{aligned}
\operatorname{Pr}\left\{\left.A\right|_{B=b}=a,\left.C\right|_{B=b}=c\right\} & =\frac{P_{A B C}(a, b, c)}{P_{B}(b)} \\
& =: P_{A C \mid B}(a, c \mid b) .
\end{aligned}
$$

It is straightforward to check that with these definitions one has the chain rule

$$
I(A: B C)=I(A: B)+I(A: C \mid B) .
$$

This implies the formula

$$
I(A: C \mid B)=H(A B)+H(B C)-H(A B C)-H(B) .
$$

Because the left hand side is by definition a convex combination of mutual informations, each of which is always nonnegative, we obtain strong subadditivity for classical distributions.

Theorem $1 I(A: C \mid B)=0$ if and only if $A$ and $C$ are conditionally independent given $B$, meaning

$$
\forall b \text { s.t. } P_{B}(b) \neq\left. 0 \quad A\right|_{B=b},\left.C\right|_{B=b} \text { are independent. }
$$


This is the case if and only if

$$
\begin{aligned}
P_{A B C}(a, b, c) & =P_{B}(b) P_{A \mid B}(a \mid b) P_{C \mid B}(c \mid b) \\
& =P_{A}(a) P_{B \mid A}(b \mid a) P_{C \mid B}(c \mid b),
\end{aligned}
$$

i.e. iff $A-B-C$ is a Markov chain in this order.

Proof. Clearly, the conditions are sufficient. Assume conversely that $I(A: C \mid B)=0$. By definition of the latter quantity, this implies that for all $b$ with $P_{B}(b) \neq 0$, $I(A: C \mid B=b)=0$. But this implies independence of $\left.A\right|_{B=b}$ and $\left.C\right|_{B=b}$. Hence, eq. (3) follows:

$$
\begin{aligned}
P_{A B C}(a, b, c) & =P_{B}(b) P_{A C \mid B}(a, c \mid b) \\
& =P_{B}(b) P_{A \mid B}(a \mid b) P_{C \mid B}(c \mid b) \\
& =P_{A}(a) P_{B \mid A}(b \mid a) P_{C \mid B}(c \mid b) .
\end{aligned}
$$

The remainder of the paper is devoted to describing the quantum mechanical generalisation of this equivalence between zero conditional mutual information, conditional independence, the Markov property and the factorization of the joint distribution given in eq. (3).

\section{RELATIVE ENTROPY}

Our approach to saturation of the strong subaddivity inequality will be via the quantum relative entropy; this quantity was defined by Umegaki 25] for two quantum states $\rho$ and $\sigma$ as

$$
S(\rho \| \sigma)=\operatorname{Tr}(\rho(\log \rho-\log \sigma))
$$

if the support of $\rho$ is contained in the support of $\sigma$, and $+\infty$ otherwise. We note that this definition generalises the familiar Kullback-Leibler divergence [8] of two probability distributions, just as von Neumann entropy generalises Shannon entropy.

For a bipartite state $\rho_{A B}$ it is straightforward to check that

$$
S\left(\rho_{A B} \| \rho_{A} \otimes \rho_{B}\right)=S\left(\rho_{A}\right)+S\left(\rho_{B}\right)-S\left(\rho_{A B}\right),
$$

and the latter quantity is abbreviated $I(A: B)$, in formal extension of the definition of Shannon's mutual information [19] to quantum states.

Example 2 Let $\left\{p(x), \rho_{x}\right\}$ be an ensemble of quantum states on $\mathcal{H}$. The Holevo quantity $\chi$ is defined as

$$
\chi\left(\left\{p(x), \rho_{x}\right\}\right)=S\left(\sum_{x} p(x) \rho_{x}\right)-\sum_{x} p(x) S\left(\rho_{x}\right) .
$$

Holevo [5] showed that this quantity is an upper bound to the mutual information between $x$ and the outcomes $y$ of any particular measurement performed on the states $\rho_{x}$.
It is easily seen that

$$
\chi\left(\left\{p(x), \rho_{x}\right\}\right)=I(A: B),
$$

with the bipartite state

$$
\rho_{A B}=\sum_{x} p(x)|x\rangle\left\langle\left. x\right|_{A} \otimes\left(\rho_{x}\right)_{B} .\right.
$$

For a tripartite state $\rho_{A B C}$ we can also consider the information $I(A: B C)$, which can be written as

$$
S\left(\rho_{A B C} \| \rho_{A} \otimes \rho_{B C}\right)=S\left(\rho_{A}\right)+S\left(\rho_{B C}\right)-S\left(\rho_{A B C}\right) .
$$

The difference between eqs. (5) and (4), which by virtue of the classical chain rule eq. (2) we might call the quantum conditional mutual information $I(A: C \mid B)$ is, therefore,

$$
\begin{aligned}
& S\left(\rho_{A B C} \| \rho_{A} \otimes \rho_{B C}\right)-S\left(\rho_{A B} \| \rho_{A} \otimes \rho_{B}\right) \\
& \quad=S\left(\rho_{A B}\right)+S\left(\rho_{B C}\right)-S\left(\rho_{A B C}\right)-S\left(\rho_{B}\right) .
\end{aligned}
$$

The right hand side here is nonnegative by strong subadditivity. (Note that this is an important theorem in the quantum case despite being an almost trivial observation classically.) The left hand side, however, can be rewritten as $S(\rho \| \sigma)-S(T \rho \| T \sigma)$, with the states $\rho=\rho_{A B C}$ and $\sigma=\rho_{A} \otimes \rho_{B C}$, and the quantum operation $T=\operatorname{Tr}_{C}$, the partial trace over $\mathcal{H}_{C}$, which as a linear map can be written as $T=\operatorname{id}_{A B} \otimes \operatorname{Tr}$.

Now a theorem of Uhlmann 24] (proved earlier by Lindblad [10] for the finite-dimensional case of interest here) says that for all states $\rho$ and $\sigma$ on a space $\mathcal{H}$, and all quantum operations $T: \mathcal{B}(\mathcal{H}) \rightarrow \mathcal{B}(\mathcal{K})$,

$$
S(\rho \| \sigma) \geq S(T \rho \| T \sigma),
$$

so Uhlmann's theorem implies strong subadditivity and we have equality in the latter if and only if there is equality in the former.

\section{THE EQUALITY CONDITION FOR RELATIVE ENTROPY}

The formulation in the previous section of strong subadditivity as a relative entropy monotonicity under a partial trace operation transforms the question for the equality conditions for the former into the same question for the latter. Note that by the very monotonicity relation, there is a "trivial" case of equality in eq. (7), namely if there exists a quantum operation $\widehat{T}$ mapping $T \rho$ to $\rho$ and $T \sigma$ to $\sigma$. In fact, this is the only case of equality:

Theorem 3 (Petz [15]) For states $\rho$ and $\sigma$,

$$
S(\rho \| \sigma)=S(T \rho \| T \sigma)
$$

if and only if there exists a quantum operation $\widehat{T}$ such that

$$
\widehat{T} T \rho=\rho, \quad \widehat{T} T \sigma=\sigma .
$$


Furthermore, on the support of $T \sigma, \widehat{T}$ can be given explicitly by the formula

$$
\widehat{T} \alpha=\sigma^{\frac{1}{2}} T^{*}\left((T \sigma)^{-\frac{1}{2}} \alpha(T \sigma)^{-\frac{1}{2}}\right) \sigma^{\frac{1}{2}},
$$

with the adjoint map $T^{*}$ of $T$ :

$$
T^{*}(X)=\sum_{i} A_{i}^{*} X A_{i}, \text { if } T(\alpha)=\sum_{i} A_{i} \alpha A_{i}^{*} .
$$

Observe that the definition of $\widehat{T}$ in eq. (8) depends on $\sigma$, thereby automatically ensuring that $\widehat{T} T \sigma=\sigma$. Sometimes, we add the subscript $\sigma$ to $\widehat{T}$ to emphasize the dependence.

Example 4 As in example2 let $\left\{p(x), \rho_{x}\right\}$ be an ensemble of states on $\mathcal{H}$ and define

$$
\rho_{A B}=\sum_{x} p(x)|x\rangle\left\langle\left. x\right|_{A} \otimes\left(\rho_{x}\right)_{B} .\right.
$$

There we observed that, with $\sigma=\rho_{A} \otimes \rho_{B}$,

$$
\chi\left(\left\{p(x), \rho_{x}\right\}\right)=S(\rho \| \sigma) .
$$

Now, let $\varphi$ be a quantum operation on $\mathcal{H}$ (which could be a measurement), and form $T=\mathrm{id}_{A} \otimes \varphi_{B}$. Then

$$
\begin{aligned}
\chi\left(\left\{p(x), \rho_{x}\right\}\right) & =S\left(\rho_{A B} \| \rho_{A} \otimes \rho_{B}\right) \\
& \geq S\left(T \rho_{A B} \| T\left(\rho_{A} \otimes \rho_{B}\right)\right) \\
& =\chi\left(\left\{p(x), \varphi \rho_{x}\right\}\right),
\end{aligned}
$$

which is (a generalistion of) the famous Holevo bound [5] in the form of a data processing relation.

Equality holds, according to theorem 3 if and only if $\widehat{T}$ of eq. (8) maps $T \rho$ to $\rho$. Note that we may assume without loss of generality that $\sigma=\rho_{A} \otimes \rho_{B}$ is strictly positive. But it is straightforward to check that

$$
\widehat{T}_{\sigma}=\widehat{\mathrm{id}}_{\rho_{A}} \otimes \widehat{\varphi}_{\rho_{B}}=\mathrm{id} \otimes \widehat{\varphi},
$$

hence we have equality in eq. (9) if and only if for all $x$, $\widehat{\varphi} \varphi \rho_{x}=\rho_{x}$.

Remark 5 In [2] the "transpose channel" $\widehat{T}$ of eq. (8), as it is called in [14], makes an appearance in a slightly different context: there a set of states is subjected to a quantum channel, and the problem is to find the best recovery map which maximises a fidelity criterion for the original states and the images of the channel output states. It was shown that the error using $\widehat{T}$ is always at most twice as the minimum error under the optimal recovery map.

\section{STRUCTURE OF STATES WITH EQUALITY}

Let $\rho_{A B C}$ be a state on $\mathcal{H}_{A} \otimes \mathcal{H}_{B} \otimes \mathcal{H}_{C}$. As we observed earlier, Uhlmann's theorem specialized to the states $\rho_{A B C}$ and $\sigma_{A B C}=\rho_{A} \otimes \rho_{B C}$, along with the map $T=\operatorname{Tr}_{C}$, states that

$$
S\left(\rho_{A B C} \| \rho_{A} \otimes \rho_{B C}\right) \geq S\left(\rho_{A B} \| \rho_{A} \otimes \rho_{B}\right) .
$$

Consequently, theorem 3 provides the condition for equality here: $\widehat{T} T \rho=\rho$. Now, because $T=\operatorname{id}_{A} \otimes R_{B C}$, with the restriction map $R_{B C}=\mathrm{id}_{B} \otimes \operatorname{Tr}_{C}$, and $\sigma$ is a tensor product, we obtain (compare example 4 ):

$$
\widehat{T}=\operatorname{id}_{A} \otimes \widehat{R},
$$

with $\widehat{R}=\widehat{R}_{\rho_{B C}}$.

Summarising, in the above monotonicity and hence in strong subadditivity we have equality if and only if

$$
\rho_{A B C}=(\mathrm{id} \otimes \widehat{R}) \rho_{A B} .
$$

We are now in a position to prove our main result:

Theorem 6 A state $\rho_{A B C}$ on $\mathcal{H}_{A} \otimes \mathcal{H}_{B} \otimes \mathcal{H}_{C}$ satisfies strong subadditivity (eq. (1)) with equality if and only if there is a decomposition of system $B$ as

$$
\mathcal{H}_{B}=\bigoplus_{j} \mathcal{H}_{b_{j}^{L}} \otimes \mathcal{H}_{b_{j}^{R}}
$$

into a direct sum of tensor products, such that

$$
\rho_{A B C}=\bigoplus_{j} q_{j} \rho_{A b_{j}^{L}} \otimes \rho_{b_{j}^{R} C},
$$

with states $\rho_{A b_{j}^{L}}$ on $\mathcal{H}_{A} \otimes \mathcal{H}_{b_{j}^{L}}$ and $\rho_{b_{j}^{R} C}$ on $\mathcal{H}_{b_{j}^{R}} \otimes \mathcal{H}_{C}$, and a probability distribution $\left\{q_{j}\right\}$.

Proof. The sufficiency of the condition is immediate. The proof of necessity will come from analysing the quantum Markov chain condition, eq. (11).

After defining the quantum operation $\varphi=\operatorname{Tr}_{C} \circ \widehat{R}$, the Markov condition gives us

$$
(\text { id } \otimes \varphi) \rho_{A B}=\rho_{A B} .
$$

Consider an operator $M$ on $\mathcal{H}_{A}$ with $0 \leq M \leq \mathbb{1}$, and define a state $\mu$ by

$$
p \mu=\operatorname{Tr}_{A}\left(\rho_{A B}(M \otimes \mathbb{1})\right), p=\operatorname{Tr}\left(\rho_{A B}(M \otimes \mathbb{1})\right) .
$$

Then, if $p \neq 0$, eq. (12) implies that $\varphi(\mu)=\mu$. Varying the operator $M$ we obtain a family $\mathbf{M}$ of states on $\mathcal{H}_{A}$ invariant under $\varphi$.

To this we can apply theorem 10 from the appendix. We obtain a decomposition

$$
\mathcal{H}_{B}=\bigoplus_{j} \mathcal{H}_{b_{j}^{L}} \otimes \mathcal{H}_{b_{j}^{R}}
$$


such that every $\mu \in \mathbf{M}$ can be written

$$
\mu=\bigoplus_{j} q_{j}(\mu) \rho_{j}(\mu) \otimes \omega_{j},
$$

with states $\rho_{j}(\mu)$ on $\mathcal{H}_{b_{j}^{L}}$ and $\omega_{j}$ on $\mathcal{H}_{b_{j}^{R}}$. This, in turn, easily implies the following structure for $\rho_{A B}$ :

$$
\rho_{A B}=\bigoplus_{j} q_{j} \rho_{A b_{j}^{L}} \otimes \omega_{b_{j}^{R}} .
$$

To see this, introduce the quantum operation

$$
P_{0}(\xi)=\bigoplus_{j} \operatorname{Tr}_{b_{j}^{R}}\left(\Pi_{j} \xi \Pi_{j}\right) \otimes \omega_{j},
$$

on $\mathcal{H}_{B}$, where $\Pi_{j}$ is the orthogonal projector onto the subspace $\mathcal{H}_{b_{j}^{L}} \otimes \mathcal{H}_{b_{j}^{R}}$ in eq. (13). (Its dual $P_{0}^{*}$ is the subalgebra projection from the appendix, where it is denoted the same way.) Then it is easy to calculate, for arbitrary operators $M$ and $N$ bounded between 0 and $\mathbb{1}$ :

$$
\begin{aligned}
\operatorname{Tr}\left(\rho_{A B}(M \otimes N)\right) & =p \operatorname{Tr}(\mu N) \\
& =p \operatorname{Tr}\left(P_{0}(\mu) N\right) \\
& =p \operatorname{Tr}\left(\mu P_{0}^{*}(N)\right) \\
& =\operatorname{Tr}\left(\rho_{A B}\left(M \otimes P_{0}^{*}(N)\right)\right) \\
& =\operatorname{Tr}\left(\left(\left(\operatorname{id} \otimes P_{0}\right) \rho_{A B}\right)(M \otimes N)\right) .
\end{aligned}
$$

By linearity, this holds for all operators in place of $M \otimes N$, so

$$
\rho_{A B}=\left(\mathrm{id} \otimes P_{0}\right) \rho_{A B},
$$

implying eq. (14), because

$$
\left(\mathrm{id} \otimes P_{0}\right) \xi=\bigoplus_{j} \operatorname{Tr}_{b_{j}^{R}}\left(\left(\mathbb{1} \otimes \Pi_{j}\right) \xi\left(\mathbb{1} \otimes \Pi_{j}^{*}\right)\right) \otimes \omega_{j} .
$$

But theorem [10] also gives information about $\varphi$ : introduce an environment $\mathcal{H}_{E}$ in state $\varepsilon$ and a unitary $U$ on $\mathcal{H}_{B} \otimes \mathcal{H}_{C} \otimes \mathcal{H}_{E}$ such that

$$
\widehat{R}(\alpha)=\operatorname{Tr}_{E}\left(U(\alpha \otimes|0\rangle\langle 0| \otimes \varepsilon) U^{*}\right),
$$

with a standard state $|0\rangle \in \mathcal{H}_{C}$. Because a further trace over $C$ gives us $\varphi$, we obtain the following form for $U$ (with $\mathcal{E}=\mathcal{H}_{C} \otimes \mathcal{H}_{E}$ ):

$$
U=\bigoplus_{j} \mathbb{1}_{\mathcal{H}_{b_{j} L}} \otimes U_{j}
$$

with $U_{j}$ a unitary on $\mathcal{H}_{b_{j}^{R}} \otimes \mathcal{E}$.

Putting eqs. (14) and (15) together, we finally get:

$$
\begin{aligned}
\rho_{A B C} & =\left(\operatorname{id}_{A} \otimes \widehat{R}\right) \rho_{A B} \\
& =\operatorname{Tr}_{E}\left(\left(\mathbb{1}_{A} \otimes U\right) \rho_{A B}\left(\mathbb{1}_{A} \otimes U^{*}\right)\right) \\
& =\bigoplus_{j} q_{j} \rho_{A b_{j}^{L}} \otimes \operatorname{Tr}_{E}\left(U\left(\omega_{b_{j}^{R}} \otimes|0\rangle\langle 0|\right) U^{*}\right) \\
& =\bigoplus_{j} q_{j} \rho_{A b_{j}^{L}} \otimes \rho_{b_{j}^{R} C},
\end{aligned}
$$

which is what we wanted to prove.

Quantum Markov states on the infinite tensor product of matrix algebras $\bigotimes_{i-\infty}^{\infty} M_{n}(\mathbb{C})^{(i)}$ were introduced by Accardi and Frigerio [1]. Let $\mathcal{A}_{m}$ be the subproduct of the factors with superscript $i \leq m$. Then $\mathcal{A}_{m} \subset \mathcal{A}_{m+1}$. A state $\rho$ of the infinite tensorproduct is called Markovian if for every integer $m$ there exists a unital completely positive mapping $T_{m, m+1}: \mathcal{A}_{m+1} \rightarrow \mathcal{A}_{m}$ which leaves the state $\rho$ (restricted to $\mathcal{A}_{m}$ ) invariant and the subalgebra $\mathcal{A}_{m-1}$ fixed. Accardi and Frigerio call the mapping $\mathcal{E}_{m, m+1}$ quasi-conditional expectation; its dual is the quantum analogue of the Markov kernel in classical probability theory.

Assume that $\mathcal{A}_{m-1}=\mathcal{B}\left(\mathcal{H}_{A}\right), M_{n}(\mathbb{C})^{(m)}=\mathcal{B}\left(\mathcal{H}_{B}\right)$ and $M_{n}(\mathbb{C})^{(m+1)}=\mathcal{B}\left(\mathcal{H}_{C}\right)$. If the equality in strong subadditivity is satisfied in this setting, then we have eq. (10) and the dual of $\widehat{T}$ is a quasi-conditional expectation. Therefore the equality in strong subadditivity for every $m$ yields a quantum Markov state on the infinite system. This property characterises quantum Markov states, see e.g. 14], p. 201.

We propose to call a state as in eq. (11) a short quantum Markov chain (as opposed to the infinite chains introduced in [1]), since we require the existence of the quasi-conditional expectation only for $\mathcal{B}\left(\mathcal{H}_{A} \otimes \mathcal{H}_{B} \otimes\right.$ $\left.\mathcal{H}_{C}\right) \rightarrow \mathcal{B}\left(\mathcal{H}_{A} \otimes \mathcal{H}_{B}\right)$; note that the analogous quasiconditional expectation $\mathcal{B}\left(\mathcal{H}_{A} \otimes \mathcal{H}_{B}\right) \rightarrow \mathcal{B}\left(\mathcal{H}_{A}\right)$ exists trivially because the subalgebra to be left invariant is $\mathbb{C}$.

Corollary 7 For a state $\rho_{A B C}$ satisfying strong subadditivity with equality:

$I(A: C \mid B)=S\left(\rho_{A B}\right)+S\left(\rho_{B C}\right)-S\left(\rho_{A B C}\right)-S\left(\rho_{B}\right)=0$,

the marginal state $\rho_{A C}$ is separable.

Conversely, for each separable state $\rho_{A C}$ there exists an extension $\rho_{A B C}$ such that $I(A: C \mid B)=0$.

Tucci 23] has given a criterion for separability based on quantum conditional mutual information. Our above result shows that in his Theorem 1 , only conditions 1 and 2 are needed, while 3 and 4 are redundant.

\section{APPLICATIONS}

Theorem 6 provides a convenient framework for synthesizing many previously known facts in quantum information theory. To illustrate the method of its application, we present a couple of special cases from the literature.

Example 8 The fundamental problem in quantum error correction is to determine when the effect of a quantum operation $\varphi$ acting on half of a pure entangled state can be perfectly reversed. Define the coherent information

$$
I_{c}(\sigma, \varphi)=S(\varphi \sigma)-S\left(\left(\operatorname{id}_{A} \otimes \varphi\right) \Phi_{\sigma}\right),
$$


where $\Phi_{\sigma}$ is any purification of $\sigma$ to system $A$. In [18] it was shown that there exists a quantum operation $\hat{\varphi}$ such that

$$
\left(\mathrm{id}_{A} \otimes \hat{\varphi} \varphi\right) \Phi_{\sigma}=\Phi_{\sigma}
$$

if and only if

$$
I_{c}(\sigma, \varphi)=S(\sigma)
$$

By the Stinespring dilatation theorem [21], we may assume that

$$
\varphi \sigma=\operatorname{Tr}_{C}\left(U_{B C}(\sigma \otimes \psi) U_{B C}^{*}\right)
$$

for a unitary operator $U_{B C}$ and pure state ancilla $\psi$ on system $C$. If we let $|\omega\rangle=\left(\mathbb{1}_{A} \otimes U_{B C}\right)\left(\left|\Phi_{\sigma}\right\rangle \otimes|\psi\rangle\right)$ then, taking mutual informations with respect to the state $\omega=$ $|\omega\rangle\langle\omega|$,

$$
\begin{aligned}
S(\sigma) & =I(A: B C)-S(A) \text { and } \\
I_{c}(\sigma, \varphi) & =I(A: B)-S(A) .
\end{aligned}
$$

Therefore, eq. (16) holds iff $I(A: B C)=I(A: B)$. By theorem [6] we can conclude that

$$
\omega=\bigoplus_{j} q_{j} \omega_{A b_{j}^{L}} \otimes \omega_{b_{j}^{R} C} .
$$

The recovery procedure $\hat{\varphi}$ given the state

$$
\left(\operatorname{id}_{A} \otimes \varphi\right) \Phi_{\sigma}=\operatorname{Tr}_{C} \omega
$$

is then obvious: first measure $j$ before preparing the state $\omega_{b_{j}^{R} C}$ on $\mathcal{H}_{b_{j}^{R}} \otimes \mathcal{H}_{C}$. Next, perform $U_{B C}^{*}$ and discard the fixed ancilla state $\psi$. The output is exactly $\Phi_{\sigma}$. As an aside, the reason that the solution to this problem was accessible without the results of the present paper is that a closer examination of the situation reveals that, because $\Phi_{\sigma}$ is pure, only the equality conditions for the usual subadditivity inequality are required in the construction of the reversal map. Strong subadditivity, in this case, is superfluous.

Example 9 Returning to our investigation of the Holevo bound from example 4 let $\left\{p(x), \rho_{x}\right\}$ be an ensemble of states on $\mathcal{H}, \rho_{A B}=\sum_{x} p(x)|x\rangle\left\langle\left. x\right|_{A} \otimes\left(\rho_{x}\right)_{B}\right.$ and $\varphi$ a quantum operation on the $B$ system. Again by the Stinespring dilation theorem, after possibly adjoining a fixed ancilla and performing a common unitary operation to all the $\rho_{x}$, we may assume without loss of generality that $B=\tilde{B} \tilde{C}$ and $\varphi=\operatorname{Tr}_{\tilde{C}}$. An application of theorem [ then gives the conditions under which

$$
\chi\left(\left\{p(x), \rho_{x}\right\}\right)=\chi\left(\left\{p(x), \varphi \rho_{x}\right\}\right) .
$$

Namely,

$$
\rho_{A \tilde{B} \tilde{C}}=\bigoplus_{j} q_{j} \sum_{x} p(x \mid j)|x\rangle\left\langle\left. x\right|_{A} \otimes\left(\rho_{x}\right)_{\tilde{b}_{j}^{L}} \otimes \omega_{\tilde{b}_{j}^{R} \tilde{C}},\right.
$$

where $p(x \mid j) q_{j}$ is the joint probability distribution for $(x, j)$ and $\omega_{\tilde{b}_{j}^{R} \tilde{C}}$ does not depend on $x$. In the special case where $\varphi$ corresponds to a measurement operation, the additional constraint $\left[\varphi \rho_{x}, \varphi \rho_{x^{\prime}}\right]=0$ must hold because the output system is classical. Given the form of $\rho_{A \tilde{B} \tilde{C}}$, this implies $\left[\left(\rho_{x}\right)_{\tilde{b}_{j}^{L}},\left(\rho_{x^{\prime}}\right)_{\tilde{b}_{j}^{L}}\right]=0$ and, in turn, that the states $\left\{\rho_{x}\right\}$ all commute. In the language of quantum information, we have found that the accessible information of an ensemble is equal to its Holevo quantity if and only if all the states in the ensemble commute. This condition for equality actually appeared in Holevo's original paper [5].

\section{DISCUSSION}

We have exhibited the explicit structure of the tripartite states $\rho_{A B C}$ which satisfy strong subadditivity with equality. Not only are they short quantum Markov chains in the sense of [1], it is even the case that the $A$ and $C$ systems are conditionally independent given $B$, in a physically meaningful sense: there is information in the $B$ system which can be obtained by a non-demolition measurement, conditioned upon which the quantum state factorises.

By specialising our result to particular types of states, we can easily recover the entropic conditions for quantum error correction and the conditions for saturation in the Holevo bound. In the general case, our theorem characterises exactly when a quantum operation preserves correlations, whether they be classical, in the form of pure entanglement, or more exotic, such as combinations of the two or even bound entanglement [6].

We left open the problem of a similar characterisation in infinite dimension (of system $B$ - infinite $A$ and $C$ are covered by our result): we only note that our method will certainly not work, as it relies ultimately on the classification of finite dimensional operator algebras. A further interesting problem could be to address the approximate case: if a state almost satisfies strong subadditivity, does it mean that its structure is close in some sense to the form of theorem [6] There might be a relation to 2] (see remark [5), where an approximate fidelity condition was studied.

\section{Acknowledgments}

We would like to thank Chris Fuchs, Mary Beth Ruskai and Ben Schumacher for helpful discussions. PH acknowledges the support of the Sherman Fairchild Foundation and the U.S. National Science Foundation through grant no. EIA-0086038. DP was partially supported by Hungarian OTKA T032662. The work of RJ and AW was supported by the U.K. Engineering and Physical Sciences Research Council. 


\section{APPENDIX A: AN OPERATOR ALGEBRAIC DERIVATION OF THE KOASHI-IMOTO THEOREM}

Let $\rho_{1}, \ldots, \rho_{K}$ be density operators on the finitedimensional Hilbert space $\mathcal{H}$. We are interested in the quantum operations (completely positive, traceperserving linear maps) $T: \mathcal{B}(\mathcal{H}) \rightarrow \mathcal{B}(\mathcal{H})$ which leave these states invariant:

$$
\forall k \quad T \rho_{k}=\rho_{k} .
$$

By possibly shrinking $\mathcal{H}$ to the minimum joint supporting subspace of the $\rho_{k}$, we may assume that the support of $\frac{1}{K} \sum_{k} \rho_{k}$ is $\mathcal{H}$, which we shall do in the following.

¿From the Stinespring dilation theorem [21] it follows that every such $T$ can be represented as

$$
T \sigma=\operatorname{Tr}_{\mathcal{E}}\left(U_{\mathcal{H} \mathcal{E}}(\sigma \otimes \varepsilon) U_{\mathcal{H} \mathcal{E}}^{*}\right)
$$

with another Hilbert space $\mathcal{E}$, a state $\varepsilon$ on it, and a unitary acting on $\mathcal{H} \otimes \mathcal{E}$.

In 7] the following result is proved by an explicit algorithmic construction:

Theorem 10 (Koashi, Imoto [7]) Associated to the states $\rho_{1}, \ldots, \rho_{K}$ there exists a decomposition of $\mathcal{H}$ as

$$
\mathcal{H}=\bigoplus_{j} \mathcal{J}_{j} \otimes \mathcal{K}_{j}
$$

into a direct sum of tensor products, such that:

1. The states $\rho_{k}$ decompose as

$$
\rho_{k}=\bigoplus_{j} q_{j \mid k} \rho_{j \mid k} \otimes \omega_{j}
$$

where $\rho_{j \mid k}$ is a state on $\mathcal{J}_{j}, \omega_{j}$ is a state on $\mathcal{K}_{j}$ (which is independent of $k)$, and $\left(q_{j \mid k}\right)_{j}$ is a probability distribution over $j$ 's.

2. For every $T$ which leaves the $\rho_{k}$ invariant, every associated unitary from eq. A2 has the form

$$
U_{\mathcal{H} \mathcal{E}}=\bigoplus_{k} \mathbb{1}_{\mathcal{J}_{j}} \otimes U_{\mathcal{K}_{j} \mathcal{E}},
$$

with unitaries $U_{\mathcal{K}_{j} \mathcal{E}}$ on $\mathcal{K}_{j} \otimes \mathcal{E}$ that satisfy

$$
\forall j \quad \operatorname{Tr}_{\mathcal{E}}\left(U_{\mathcal{K}_{j} \mathcal{E}}\left(\omega_{j} \otimes \varepsilon\right) U_{\mathcal{K}_{j} \mathcal{E}}^{*}\right)=\omega_{j}
$$

The purpose of this appendix is to present a short (but non-constructive) proof of this theorem, based on the theory of operator algebras. Property 1 of the theorem has previously appeared in a paper of Lindblad [12] and our approach closely follows the one taken there.

We begin with a slight reformulation of the result, avoiding the environment system $\mathcal{E}$ :
Proposition 11 In the above theorem, property 2 is equivalent to

$2^{\prime}$. For every $T$ which leaves the $\rho_{k}$ invariant,

$$
\left.\forall j \quad T\right|_{\mathcal{B}\left(\mathcal{J}_{j} \otimes \mathcal{K}_{j}\right)}=\mathrm{id} \otimes T_{j},
$$

with id on $\mathcal{J}_{j}$ and $T_{j}$ on $\mathcal{K}_{j}$ such that $T_{j}\left(\omega_{j}\right)=\omega_{j}$.

Proof. Clearly, 2 implies $2^{\prime}$. In the other direction, consider any $U$ implementing $T$. Clearly, because of $2^{\prime}$,

$$
\left.U\right|_{\left(\mathcal{J}_{j} \otimes \mathcal{K}_{j}\right) \mathcal{E}}=\mathbb{1}_{\mathcal{J}_{j}} \otimes U_{\mathcal{K}_{j} \mathcal{E}},
$$

which yields the form 2 for $U$.

Proof of theorem [10. Consider the set of quantum operations

$$
\mathbf{F}=\left\{F: \forall k F \rho_{k}=\rho_{k}\right\},
$$

which is obviously non-empty since it contains $T$ and id.

With each $F \in \mathbf{F}$ we associate the set

$$
\mathcal{A}_{F}=\left\{X \in \mathcal{B}(\mathcal{H}): F^{*}(X)=X\right\}
$$

of operators left invariant by the adjoint map $F^{*}$. By lemma 12 this is a $*$-subalgebra of $\mathcal{B}(\mathcal{H})$ and, in fact, if $F^{*}(X)=\sum_{i} B_{i}^{*} X B_{i}$,

$$
\mathcal{A}_{F}=\left\{B_{i}, B_{i}^{*}\right\}^{\prime}=\left\{X: \forall i X B_{i}=B_{i} X, X B_{i}^{*}=B_{i}^{*} X\right\}
$$

is the commutator of the Kraus operators of $F^{*}$. By the same lemma, this algebra furthermore is the image of $\mathcal{B}(\mathcal{H})$ under the projection map

$$
P^{*}=\lim _{N \rightarrow \infty} \frac{1}{N} \sum_{n=1}^{N}\left(F^{*}\right)^{n},
$$

whose adjoint is

$$
P=\lim _{N \rightarrow \infty} \frac{1}{N} \sum_{n=1}^{N} F^{n} .
$$

Clearly, $P \in \mathbf{F}$. Next, define

$$
\mathcal{A}_{0}=\bigcap_{F \in \mathbf{F}} \mathcal{A}_{F},
$$

which clearly is a $*$-subalgebra itself. Because all dimensions are finite, it can actually be presented as a finite intersection

$$
\mathcal{A}_{0}=\mathcal{A}_{F_{1}} \cap \ldots \cap \mathcal{A}_{F_{M}},
$$

and in fact there is $F_{0} \in \mathbf{F}$ such that $\mathcal{A}_{0}=\mathcal{A}_{F_{0}}$. We may take, for example,

$$
F_{0}=\frac{1}{M} \sum_{\mu=1}^{M} F_{\mu}
$$

and use lemma 12 Denote the projection onto $\mathcal{A}_{0}$ derived from $F_{0}^{*}$ by $P_{0}^{*}$. 
Lemma 13 gives us the form of $\mathcal{A}_{0}$ :

$$
\mathcal{A}_{0}=\bigoplus_{j} \mathcal{B}\left(\mathcal{H}_{b_{j}^{L}}\right) \otimes \mathbb{1}_{b_{j}^{R}}
$$

and, likewise, of $P_{0}^{*}$ :

$$
P_{0}(\xi)=\bigoplus_{j} \operatorname{Tr}_{b_{j}^{R}}\left(\Pi_{j} \xi \Pi_{j}\right) \otimes \omega_{j} .
$$

Thus, we obtain the advertised form of the states:

$$
\rho_{k}=P_{0}\left(\rho_{k}\right)=\bigoplus_{j} q_{j} \rho_{j \mid k} \otimes \omega_{j}
$$

As for the properties of $T$, because $\mathcal{A}_{T} \supset \mathcal{A}_{0}$, we have $\left.T^{*}\right|_{\mathcal{A}_{0}}=\operatorname{id}_{\mathcal{A}_{0}}$. More explicitly, for $A \in \mathcal{B}\left(\mathcal{J}_{j}\right)$ and $\mathbb{1} \in$ $\mathcal{B}\left(\mathcal{K}_{j}\right)$,

$$
T^{*}(A \otimes \mathbb{1})=A \otimes \mathbb{1} .
$$

Now assume $0 \leq A \leq \mathbb{1}$, and consider $B \in \mathcal{B}\left(\mathcal{K}_{j}\right)$ such that $0 \leq B \leq \mathbb{1}$. Then

$$
0 \leq T^{*}(A \otimes B) \leq T^{*}(A \otimes \mathbb{1})=A \otimes \mathbb{1} \leq \mathbb{1} \otimes \mathbb{1} .
$$

This implies that $T^{*}$ maps $\mathcal{B}\left(\mathcal{J}_{j} \otimes \mathcal{K}_{j}\right)$ into itself for all $j$, and hence the same applies to $T$.

Now, eq. A4 applied with the rank-one projector $A=$ $|\psi\rangle\langle\psi|$, yields that

$$
T^{*}(|\psi\rangle\langle\psi| \otimes B)=|\psi\rangle\langle\psi| \otimes B^{\prime},
$$

with $B^{\prime}$ depending linearly on $B$. Dependence on $|\psi\rangle\langle\psi|$ quickly leads to contradiction, so

$$
T^{*}(A \otimes B)=A \otimes T_{j}^{*}(B),
$$

which gives the desired form of $T$ :

$$
T(\rho \otimes \sigma)=\rho \otimes T_{j}(\sigma),
$$

and application to the $\rho_{k}$ yields the invariance of the $\omega_{j}$ under $T_{j}$.

Here follow the general lemmas about unital completely positive maps which were used in the proof of theorem 10] The first one is a mean ergodic theorem for the dual of a quantum operation. (The statement is essentially the Kovács-Szücs theorem - see e.g. [4], proposition 4.3.8 - but we give a proof in our setting.)

Lemma 12 For a quantum operation $F$, the map

$$
P^{*}=\lim _{N \rightarrow \infty} \frac{1}{N} \sum_{n=1}^{N}\left(F^{*}\right)^{n}
$$

is a conditional expectation onto the $*$-subalgebra

$$
\mathcal{A}_{F}=\left\{X: F^{*}(X)=X\right\}=\left\{B_{i}, B_{i}^{*}\right\}^{\prime} .
$$

Proof. First of all, we want to see that $\mathcal{A}_{F}$ is a ${ }^{*-}$ subalgebra. It is a linear subspace and the Kraus representation shows that if $F^{*}(X)=X$, then $F^{*}\left(X^{*}\right)=$ $\left(F^{*}(X)\right)^{*}=X^{*}$.
With this, the Schwarz inequality (see e.g. [4]) gives that for invariant $X$,

$$
F^{*}\left(X^{*} X\right) \geq F^{*}\left(X^{*}\right) F^{*}(X)=X^{*} X .
$$

However, applying a faithful (i.e., non-degenenerate) invariant state, such as $\frac{1}{K} \sum_{k} \rho_{k}$, leaves only the possibility of equality:

$$
F^{*}\left(X^{*} X\right)=X^{*} X,
$$

from which it follows straightforwardly that the product of invariant operators is again invariant.

For $X \in \mathcal{A}_{F}$, one can confirm by direct calculation that

$$
\sum_{i}\left[X, B_{i}\right]^{*}\left[X, B_{i}\right]=F^{*}\left(X^{*} X\right)-X^{*} X=0,
$$

the latter by the previous observation that $X^{*} X$ is also invariant. But since the left hand side is a sum of positive terms, all of them must be 0 , hence $\left[X, B_{i}\right]=0$ for all $i$. Similarly, $\left[X, B_{i}^{*}\right]=0$ for all $i$.

These facts together say that $\mathcal{A}_{F} \subset\left\{B_{i}, B_{i}^{*}\right\}^{\prime}$, while the opposite containment is trivial.

Another application of the Schwarz inequality gives that $F^{*}$ is a contraction. Hence the mean ergodic theorem for a contraction implies that the limit in the statement exists. (Due to the finite dimensional situation all the relevant topologies coincide.) To see $P^{*}(X) \in \mathcal{A}_{F}$, we compute

$\left(F^{*} P^{*}\right)(X)-P^{*}(X)=\lim _{N \rightarrow \infty} \frac{1}{N}\left(\left(F^{*}\right)^{N+1}(X)-F^{*}(X)\right)$,

which is clearly 0 so the image of $P^{*}$ is contained in $\mathcal{A}_{F}$. Since $P^{*}(X)=X$ when $X \in \mathcal{A}_{F}$, it is also onto and a projection.

Lemma 13 Let $\mathcal{A}$ be a $*$-subalgebra of $\mathcal{B}(\mathcal{H})$, with a finite dimensional $\mathcal{H}$. Then there is a direct sum decomposition

$$
\mathcal{H}=\bigoplus_{j} \mathcal{H}_{b_{j}^{L}} \otimes \mathcal{H}_{b_{j}^{R}}
$$

such that

$$
\mathcal{A}=\bigoplus_{j} \mathcal{B}\left(\mathcal{H}_{b_{j}^{L}}\right) \otimes \mathbb{1}_{b_{j}^{R}}
$$

Any completely positive and unital projection $P^{*}$ of $\mathcal{B}(\mathcal{H})$ onto $\mathcal{A}$ is of the form

$$
P^{*}(X)=\bigoplus_{j} \operatorname{Tr}_{b_{j}^{R}}\left(\Pi_{j} X \Pi_{j}\left(\mathbb{1}_{b_{j}^{L}} \otimes \omega_{j}\right)\right) \otimes \mathbb{1}_{b_{j}^{R}},
$$

with the projections $\Pi_{j}$ onto the subspaces $\mathcal{H}_{b_{j}^{L}} \otimes \mathcal{H}_{b_{j}^{R}}$, and states $\omega_{j}$ on $\mathcal{H}_{b_{j}^{R}}$.

Proof. See [22], section I.11. 
[1] L. Accardi, A. Frigerio, "Markovian cocycles", Proc. Proc. Roy. Irish Acad., vol. 83A, no. 2, pp. 251-263, 1983.

[2] H. Barnum, E. Knill, "Reversing quantum dynamics with near-optimal quantum and classical fidelity", J. Math. Phys., vol. 43, no. 5, pp. 2097-2106, 2002.

[3] H. Barnum, M. A. Nielsen, B. Schumacher, "Information transmission through a noisy quantum channel", Phys. Rev. A, vol. 57, no. 6, pp. 4153-4175, 1998.

[4] O. Bratteli, D. W. Robinson, Operator algebras and quantum statistical mechanics. 1. $C^{*}$ - and $W^{*}$-algebras, symmetry groups, decomposition of states, 2nd ed., Texts and Monographs in Physics, Springer Verlag, New York, 1987.

[5] A. S. Holevo, "Bounds for the quantity of information transmitted by a quantum channel", Probl. Inf. Transm., vol. 9, no. 3, pp. 177-183, 1973.

[6] M. Horodecki, P. Horodecki, R. Horodecki, "Mixed-state entanglement and distillation: Is there a 'bound' entanglement in nature?", Phys. Rev. Lett. vol. 80, pp. 52395242,1998

[7] M. Koashi, N. Imoto, "Operations that do not disturb partially known quantum states", Phys. Rev. A, vol. 66, no. 2, 022318, 2002.

[8] S. Kullback, R. A. Leibler, "On information and sufficiency", Ann. Math. Statistics, 1951.

[9] E. H. Lieb, M. B. Ruskai, "Proof of the strong subadditivity of quantum-mechanical entropy", J. Math. Phys., vol. 14, pp. 1938-1941, 1973.

[10] G. Lindblad, "Completely positive maps and entropy inequalities", Comm. Math. Phys., vol. 40, 147-151, 1975.

[11] G. Lindblad, "Quantum entropy and quantum measurements", in: C. Bendjaballah, O. Hirota, S. Reynaud (eds.), Quantum Aspects of Optical Communications, Lecture Notes in Physics, vol. 378, pp. 71-80, Springer Verlag, Berlin, 1991.

[12] G. Lindblad, "A general no-cloning theorem", Lett. Math. Phys, vol. 47, pp. 189-196, 1999.

[13] J. von Neumann, "Thermodynamik quantenmechanischer Gesamtheiten", Nachr. der Gesellschaft der Wiss. Gött., pp. 273-291, 1927. (See also J. von Neumann, Mathematical Foundations of Quantum Mechan- ics, Princeton University Press, Princeton, NJ, 1996.)

[14] M. Ohya, D. Petz, Quantum Entropy and Its Use, Springer Verlag: Texts and Monographs in Physics, Berlin Heidelberg, 1993.

[15] D. Petz, "Sufficient subalgebras and the relative entropy of states of a von Neumann algebra", Comm. Math. Phys., vol. 105, no. 1, pp. 123-131, 1986. "Sufficiency of channels over von Neumann algebras", Quart. J. Math. Oxford Ser. (2), vol. 39, no. 153, pp. 97-108, 1988.

[16] D. Petz, "Monotonicity of quantum relative entropy revisited", Rev. Math. Phys., vol. 15, pp. 79-91, 2003.

[17] M. B. Ruskai, "Inequalities for Quantum Entropy: A Review with Conditions for Equality", J. Math. Phys., vol. 43, pp. 4358-4375, 2002.

[18] B. Schumacher, M. A. Nielsen, "Quantum data processing and error correction", Phys. Rev. A., vol. 54, pp. 2629-2635, 1996.

[19] C. E. Shannon, "A mathematical theory of communication", Bell Syst. Tech. Journal, vol. 27, pp. 379-423, 623-656, 1948.

[20] P. W. Shor, "Additivity of the Classical Capacity of Entanglement-Breaking Quantum Channels", J. Math. Phys., vol. 43, pp. 4334-4340, 2002.

[21] W. F. Stinespring, "Positive functions on $\mathrm{C}^{*}$-algebras", Proc. Amer. Math. Soc., vol. 6, pp. 211-216, 1955.

[22] M. Takesaki, Theory of Operator Algebras I, SpringerVerlag, New York-Heidelberg-Berlin, 1979.

[23] R. R. Tucci, "Separability of Density Matrices and Conditional Information Transmission", e-print quant-ph/0005119 2000. Based on "Quantum Entanglement and Conditional Information Transmission", eprint quant-ph/9909041, 1999.

[24] A. Uhlmann, "Relative entropy and the WignerYanase-Dyson-Lieb concavity in an interpolation theory", Comm. Math. Phys., vol. 54, no. 1, pp. 21-32, 1977.

[25] H. Umegaki, "Conditional expectation in an operator algebra IV. Entropy and information", Kōdai Math. Sem. Rep., vol. 14, pp. 59-85, 1962.

[26] A. Wehrl, "General properties of entropy", Rev. Modern Phys., vol. 50, no. 2, pp. 221-260, 1978. 